Journal for ImmunoTherapy of Cancer

\section{checkpoint inhibitor therapy are at an increased risk for atherosclerotic cardiovascular disease}



Esther Lutgens, ${ }^{1,2,3}$ Tom T.P. Seijkens ${ }^{1}$
To cite: Lutgens $\mathrm{E}$, Seijkens TT.P. Cancer patients receiving immune checkpoint inhibitor therapy are at an increased risk for atherosclerotic cardiovascular disease. Journal for Immunotherapy of Cancer 2020;8:e000300. doi:10.1136/ jitc-2019-000300

Accepted 22 January 2020
Check for updates

(c) Author(s) (or their employer(s)) 2020. Re-use permitted under CC BY-NC. No commercial re-use. See rights and permissions. Published by BMJ.

${ }^{1}$ Department of Medical Biochemistry, Amsterdam Cardiovascular Sciences (ACS), Amsterdam University Medical Centers, University of Amsterdam, Amsterdam, The Netherlands

${ }^{2}$ Institute for Cardiovascular Prevention (IPEK), Ludwig Maximilian's University, Munich, Germany

${ }^{3}$ German Centre for Cardiovascular Research (DZHK), partner site Munich Heart Alliance, Munich, Germany

Correspondence to

Dr Tom T.P. Seijkens;

t.t.seijkens@amsterdamumc.nl

\section{ABSTRACT}

The widespread clinical use of immune checkpoint inhibitors (ICl) has increased our knowledge on their adverse effects on chronic inflammatory diseases. Atherosclerosis, a low-grade lipid-driven inflammatory disease of the larger arteries, is commonly present in cancer patients. A major concern is the adverse effect of ICI on atherosclerosis-related cardiovascular disease, resulting in cardiovascular events, such as myocardial infarction or ischaemic stroke. The effects of ICl on atherosclerosis in cancer patients are incompletely understood, but it is well known that immune checkpoint proteins orchestrate the inflammatory response underlying atherogenesis. This paper addresses the hypothesis that $\mathrm{ICl}$ therapy puts cancer patients at an increased risk for atherosclerosis-related cardiovascular disease, that might only become apparent years after ICI therapy. Until clinical and experimental studies have addressed this hypothesis, optimal cardiovascular risk management in ICl-treated patients is opportune to reduce the occurrence of cardiovascular disease in cancer patients and long-term cancer survivors.

The growing clinical use of immune checkpoint inhibitors (ICI) has increased our knowledge on the immune-related adverse events (IRAE) affecting the cardiovascular system. ${ }^{1}$ Myocarditis, arrhythmia, and cardiomyopathy are the most frequently described cardiovascular IRAE, affecting 1\%-1.5\% of the patients that receive ICI therapy. ${ }^{1}$ The increased awareness of cardiovascular IRAE stimulates initiatives to improve the care for cancer patients who develop these toxicities, such as practice guidelines, clinical cardio-oncology programs, clinical registries, and the launch of focused scientific journals. ${ }^{1}$ These important initiatives will undoubtedly increase our knowledge on cardiovascular IRAE and stimulate the development of evidence-based treatment strategies for these potentially lethal complications. ${ }^{1}$ The effects of ICI on more gradually developing cardiovascular diseases, in particular atherosclerosis, are relatively underexposed in these promising initiatives. From a vascular point of view, we would like to discuss some considerations on the effects of ICI on atherosclerosisrelated cardiovascular disease. This paper addresses the hypothesis that ICI therapy aggravates atherosclerosis, thereby provoking more common cardiovascular diseases and events, such as myocardial infarction, peripheral arterial disease, and ischemic stroke, in cancer patients.

Atherosclerosis is a chronic lipid-driven inflammatory disease that results in the formation of lipid-rich and immune cellrich plaques in the arterial wall. ${ }^{2}$ During the progression of atherosclerosis, these lesions may rupture, which results in thrombus formation and subsequent vascular occlusion. ${ }^{2}$ Single-cell RNA sequencing as well as mass cytometry of human atherosclerotic plaques recently demonstrated that $\mathrm{T}$ cells are a dominant immune cell type in human atherosclerotic lesions. ${ }^{3}$ Both $\mathrm{CD} 4^{+}$and $\mathrm{CD} 8^{+} \mathrm{T}$ cells in the plaque display an activated profile, which will not only promote the initiation of atherosclerotic lesion formation but also drives the progression towards vulnerable plaques that may trigger myocardial infarction or ischemic stroke on rupture. ${ }^{23}$ It is well known that immune checkpoint proteins orchestrate the inflammatory response that underlies atherogenesis and preclinical studies have elucidated the role of the ICI targets cytotoxic T-lymphocyte associated protein 4 (CTLA4) and programmed cell death protein 1 (PD1) in atherosclerosis. ${ }^{2}$ For example, $T$ cell-specific overexpression of CTLA4 reduces atherosclerotic lesion formation in apolipoprotein $\mathrm{E}$ deficient mice and limits plaque inflammation, as reflected by decreased $\mathrm{CD} 4^{+} \mathrm{T}$ cells and macrophage abundance. ${ }^{2}$ While CTLA4 overexpression reduced systemic regulatory $\mathrm{T}$ cell numbers, the suppressive capacity of these cells increased and $\mathrm{CD} 4^{+} \mathrm{T}$ cell proliferation, activation, and cytokine production was 
reduced, resulting in an atheroprotective $\mathrm{T}$ cell profile in hyperlipidemic mice. ${ }^{2}$ Accordingly, antibody-mediated blockage of CTLA4 aggravated postinterventional lesion formation in atherosclerotic mice by increasing plaque $\mathrm{T}$ cell abundance. ${ }^{2}$ Pharmacological modulation of CTLA4 interactions by the CTLA4-Ig fusion protein abatacept, which prevents CD28-CD80/86-mediated immune cell activation, reduced hyperhomocysteinemia-accelerated atherosclerosis by hampering $\mathrm{T}$ cell-driven responses. ${ }^{2} \mathrm{~A}$ similar anti-atherogenic role has been attributed to the PD1-PDL1 dyad as genetic deficiency of PD1 aggravates atherosclerosis in hyperlipidemic mice by increasing $\mathrm{CD} 4^{+}$ and $\mathrm{CD}^{+} \mathrm{T}$ cell effector functions and their abundance in plaques. ${ }^{2}$ These studies convincingly demonstrate that CTLA4 and PD1 put a brake on T cell-driven inflammation in experimental atherosclerosis, thereby hampering plaque development and progression.

Subclinical atherosclerosis is a common phenomenon and is found in $45 \%-75 \%$ of the patients with cancer. ${ }^{4}$ As cardiovascular disease and cancer have multiple shared risk factors, including ageing, physical inactivity, tobacco use, and chronic low grade inflammation, cancer patients may have an increased risk to develop clinical complications of atherosclerosis, such as myocardial infarction or ischemic stroke. ${ }^{4}$ Clinical data on the effects of ICI on atherosclerotic cardiovascular disease are still sparse and it is very likely that the effects of ICI on atherosclerosis have been underestimated so far as the elderly, who more often have subclinical atherosclerosis, and patients with a history of cardiovascular disease were excluded from most of the clinical trials investigating effects of ICI on cancer. Moreover, atherosclerosis-related complications develop gradually over years or decades, as ICI have been implemented in the clinic in the past decade, the longterm effects of ICI are hardly known. Nevertheless, accumulating studies report atherosclerosis-related acute vascular events in ICI-treated patients. ${ }^{56}$ For example, a meta-analysis of 22 trials targeting PD1-PDL1 in patients with lung cancer reported that myocardial infarction or ischemic stroke occurred in $3 \%$ of the patients. ${ }^{7}$ In addition, Bar and colleagues retrospectively identified the occurrence of acute vascular events in 1215 ICI-treated patients with non-small cell lung cancer. ${ }^{5}$ Approximately $1 \%$ of the patients in this cohort developed a myocardial infarction or stroke within the first 6 months after initiation of ICI therapy, suggesting that ICI-related vascular events resulted from effects on existing atherosclerotic plaques rather than on de novo lesion formation. ${ }^{5}$ Although the optimal duration of ICI therapy is uncertain, these clinical observations suggest that even short-term ICI treatment may provoke atherosclerotic cardiovascular disease. Whether a longer duration of treatment further increases the risk for these adverse effects is currently not known. Rapid progression of atherosclerosis has also been observed in a patient with a giant cell bone tumor who developed two subsequent myocardial infarctions after ICI therapy. ${ }^{8}$ After the first myocardial infarction, coronary angiography showed only minimal atherosclerosis in the left circumflex, when the patients experienced recurrent chest pain 2 months after the initial presentation, significant stenosis of the proximal circumflex was observed, reflecting rapid progression or rupture of a pre-existing atherosclerotic lesions. ${ }^{8}$ In line with observations in atherosclerotic mice, these studies suggest that ICI directly affect atherosclerotic plaque inflammation, thereby promoting the progression of atherosclerotic lesions towards clinically unfavorable unstable lesions.

A recent autopsy study in 11 cancer patients, who died from non-cardiovascular causes, provides additional insights in the pathophysiology of ICI therapy-associated atherosclerosis. Anti-PD(L) 1 therapy or combined antiPD(L) 1 and anti-CTLA4 therapy altered the immune cell composition in coronary atherosclerotic lesions, resulting in an increased CD3/CD68 ratio. ${ }^{9}$ The mechanisms underlying the altered immune cell composition in plaques were not investigated in this study, but ICI-induced T cell infiltration into the plaque, (re-)activation of plaque $\mathrm{T}$ cells or increased $\mathrm{T}$ cell-induced macrophage apoptosis may have contributed to the increased CD3/CD68 ratio. Regardless of underlying pathophysiological mechanisms, this study demonstrates that ICI therapy induces a $\mathrm{T}$ cell-driven inflammatory response in human atherosclerotic plaques, which is in contrast to a macrophagedriven inflammatory responses that is commonly found in atherosclerotic lesions. ${ }^{9}$ Importantly, a high CD3/ CD68 ratio is associated with plaque instability, potentially putting ICI-treated patients at an increased risk for myocardial infarction or ischemic stroke. ${ }^{9}$

Although larger and long-term follow-up studies are required to determine the exact incidence of acute vascular events in ICI-treated patients, several lessons can be learnt from the studies that have been reported so far. First, the majority of ICI-treated patients who developed acute vascular complications had at least two conventional cardiovascular risk factors, such as dyslipidemia, hypertension, obesity, a history of smoking or a previous cardiovascular event. ${ }^{5}$ Second, atherosclerosis-related adverse events appear not to be correlated with the occurrence of other IRAE. ${ }^{5}$ Third, the occurrence of acute vascular events in ICI-treated patients significantly reduced the overall survival of these patients and $25.8 \%$ of the patients died within 4 weeks after the event, suggesting that prevention of these adverse atherosclerosis-related cardiovascular events improves the prognosis and qualityof-life of ICI-treated patients. ${ }^{5}$ Another important point is the potential interaction between cardiovascular risk management and ICI therapy. For example, simvastatin, a $\beta$-hydroxy $\beta$-methylglutaryl-CoA (HMG-CoA) reductase inhibitor that is commonly used for the treatment of dyslipidemia, improved the efficacy of anti-PD1 therapy in experimental tumor models by directly enhancing anti-tumor $\mathrm{T}$ cell responses, suggesting that conventional cardiovascular drugs may affect the efficacy of ICI therapy, an important concept that warrants further investigation. ${ }^{10}$ 
ICI are becoming standard of care for multiple types of cancer and novel combinational ICI strategies are increasingly applied, also in patients with a history of cardiovascular disease, the elderly and patients who previously received or concurrently receive chemotherapy and/or radiotherapy, which may also affect the atherosclerotic risk. Until clinical and experimental studies have elucidated the effects of ICI on atherosclerosis, optimal cardiovascular risk management and cardiovascular disease surveillance in cancer patients and longterm cancer survivors may be opportune to reduce the risk for ICI-associated acute vascular events, especially as atherosclerosis-related adverse events severely compromise the quality-of-life as well as morbidity and mortality rates of these patients.

Acknowledgements We apologize to authors whose work could not be cited due to space limitations.

Contributors EL and TS contributed to the design and drafting of the manuscript.

Funding This work was supported by the Netherlands Heart Institute (Young@ Heart grant to TS), Amsterdam Cardiovascular Sciences (MD/PhD grant to TS), the Dutch Heart Foundation (clinical scientist grant to TS), The Netherlands CardioVascular Research Initiative: the Dutch Heart Foundation, Dutch Federation of University Medical Centers, the Netherlands, Organization for Health Research and Development, and the Royal Netherlands Academy of Sciences for the GENIUS-II project 'Generating the best evidence-based pharmaceutical targets for atherosclerosis-II' (CVON2018-19). This study was also supported by the Netherlands Organization for Scientific Research (NWO) (VICI grant 016.130.676 to EL), the EU (H2020-PHC-2015-667673, REPROGRAM to EL), the European Research Council (ERC consolidator grant CD40-INN 681492 to EL), and the German Science Foundation (DFG, CRC1123, project A5).

Competing interests None declared.
Patient consent for publication Not required.

Provenance and peer review Not commissioned; externally peer reviewed.

Open access This is an open access article distributed in accordance with the Creative Commons Attribution Non Commercial (CC BY-NC 4.0) license, which permits others to distribute, remix, adapt, build upon this work non-commercially, and license their derivative works on different terms, provided the original work is properly cited, appropriate credit is given, any changes made indicated, and the use is non-commercial. See http://creativecommons.org/licenses/by-nc/4.0/.

\section{REFERENCES}

1 Ball S, Ghosh RK, Wongsaengsak S, et al. Cardiovascular toxicities of immune checkpoint inhibitors: JACC review topic of the week. $J$ Am Coll Cardiol 2019;74:1714-27.

2 Seijkens TTP, van Tiel CM, Kusters PJH, et al. Targeting CD40induced TRAF6 signaling in macrophages reduces atherosclerosis. $J$ Am Coll Cardiol 2018;71:527-42.

3 Fernandez DM, Rahman AH, Fernandez NF, et al. Single-Cell immune landscape of human atherosclerotic plaques. Nat Med 2019;25:1576-88.

4 Vincent L, Leedy D, Masri SC, et al. Cardiovascular disease and cancer: is there increasing overlap? Curr Oncol Rep 2019;21:47.

5 Bar J, Markel G, Gottfried T, et al. Acute vascular events as a possibly related adverse event of immunotherapy: a single-institute retrospective study. Eur J Cancer 2019;120:122-31.

6 Tomita Y, Sueta D, Kakiuchi Y, et al. Acute coronary syndrome as a possible immune-related adverse event in a lung cancer patient achieving a complete response to anti-PD-1 immune checkpoint antibody. Ann Oncol 2017;28:2893-5.

$7 \mathrm{Hu}$ Y-B, Zhang Q, Li H-J, et al. Evaluation of rare but severe immune related adverse effects in PD-1 and PD-L1 inhibitors in non-small cell lung cancer: a meta-analysis. Trans/ Lung Cancer Res 2017;6:S8-20.

8 Kwan JM, Cheng R, Feldman LE. Hepatotoxicity and recurrent NSTEMI while on pembrolizumab for metastatic giant cell bone tumor. Am J Med Sci 2019;357:343-7.

9 Newman JL, Stone JR. Immune checkpoint inhibition alters the inflammatory cell composition of human coronary artery atherosclerosis. Cardiovasc Pathol 2019;43:107148.

$10 \mathrm{Xia}$ Y, Xie Y, Yu Z, et al. The mevalonate pathway is a druggable target for vaccine adjuvant discovery. Cell 2018;175:1059-73. 